\title{
EFFECTS OF THOULET'S REAGENT ON ACTOMYOSIN AND ITS COMPONENT PROTEINS*
}

\author{
TORAO NAGAI, MADOKA MAKINOSE \\ AND TAKASHI OCHIAI** \\ Department of Physiology, Sapporo Medical College, Sapporo, Japan
}

Varga (1) once reported that the glycerinated muscle fiber can be contracted not only by adenosinetriphosphate (ATP) but also by some other agents, such as $\mathrm{K}_{2} \mathrm{HgI}_{4}, \mathrm{CuCl}_{2}$ and heat. On the other hand, the contraction of myosin thread by heat denaturation was reported by Weber in former times (2). Lately, Laki and Bowen $(3,4)$ studied the effects of $\mathrm{K}_{2} \mathrm{HgI}_{4}$ (Thoulet's reagent) on actomyosin thread and glycerinated muscle fiber and they reported that this reagent can induce contraction which is almost the same as that induced by ATP. Morales attended seriously to these results and stated in his report (5) that these results raised a real question about the nature of ATP contraction of the glycerinated muscle fiber.

The present authors studied previously the effect of Thoulet's reagent on the glycerinated muscle fiber in detail, and compared this effect with those of ATP, $\mathrm{CuCl}_{2}$ and heat (6). They observed that Thoulet's reagent contraction differs from ATP contraction in regard to the influence of many factors on the contraction while the effect of the reagent bears considerable resemblance to that of $\mathrm{CuCl}_{2}$ and heat. Then they concluded that the contraction of glycerinated muscle fiber induced by Thoulet's reagent may be due to the irreversible denaturation of the actomyosin and that the contraction by the reagent differs in nature from that by ATP concerning the mechanism of contraction.

In this report, the effect of Thoulet's reagent on actomyosin (AM) and its component proteins, i.e. myosin, actin, $\mathrm{H}$ - and L-meromyosin, was studied and was compared with that of ATP in order to clarify further the difference between the effects of both agents, Thoulet's reagent and ATP, on muscle models.

\section{EXPERIMENTAL}

1) Materials: Myosin-B and crystalline myosin were prepared from rabbit muscle following the method of Szent-Györgyi (7). Myosin-B was washed three times as described by Edsall (8).

Received for publication June 29, 1957.

* A preliminary report was published on Journal of Medical Science (in Japanese), Igaku-Shoin, Tokyo, October-December, 1956. The essential points of this paper were presented at the meeting of Physiological Society of Japan, Okayama, May, 1956.

** 永井寅男, 牧之瀬 望, 落合 倜 
L- and H-meromyosin were prepared following the method of A. G. SzentGyörgyi (9). L-meromyosin which was separated from $\mathrm{H}$-meromysin by dialysis and centrifugation was dissolved in $0.6 \mathrm{M} \mathrm{KCl}$ solution and was reprecipitated by addition of 10 volumes of deionized water. The precipitate was dissolved again in $0.6 \mathrm{M} \mathrm{KCl}$ solution.

F-actin was prepared following the method of Straub (10).

2) Test solutions: Thoulet's reagent, ATP, $\mathrm{CuSO}_{4}$ and $\mathrm{KI}$ were used.

In order to keep $p_{\mathrm{H}}$ of the reaction mixture constant, $0.057 \mathrm{~m}$ Michaelis veronal-acetate buffer ( $\left.p_{\mathrm{H}} 7\right)$ was used.

3) Reaction mixtures: The whole quantity of each mixture was $2 \mathrm{cc}$. The composition of the mixture was shown in each illustration of the figures. The concentration of each component in the illustrations means the final value.

4) Observations: Firstly, the reaction mixture without the test solution was incubated 10 minutes in the water bath at $20^{\circ} \mathrm{C}$. before the addition of the test solution. Then the reaction was started by the addition of the test solution.

The grade of the precipitation of myosin- $B$ induced by the Thoulet's reagent is represented by the value of $\mathrm{A} / \mathrm{A}_{0} \times 100(\%)$ ( $\mathrm{A}$ : the height of the supernatant column of the precipitated $\mathrm{AM}$ in a given concentration of $\mathrm{KCl}$ or Thoulet's reagent. $A_{0}$ : the height of the supernatant column of the precipitated $A M$ in maximum precipitation).

\section{RESULTS}

Thoulet's reagent induces the isodimensional syneresis of AM (myosin-B) sol under certain condition (fig. 1). This syneresis takes place after the latent period of 2-3 minutes and proceeds so slowly that it takes $20-30$ minutes to complete the "plug-formation" of AM. This plug is yellowish, homogeneous, viscid and jelly-like. This phenomenon can be observed even at $0^{\circ} \mathrm{C}$. as well as at $20^{\circ} \mathrm{C}$. So-called "clearing phase" which appears in the process of superprecipitation can not be observed in the course of this phenomenon.

In fig. 1, the effect of Thoulet's reagent on AM is compared with the effects of $\mathrm{ATP}, \mathrm{CuSO}_{4}$ and $\mathrm{KI}$. $\mathrm{CuSO}_{4}$ induces rapidly white and gross precipitation followed by isodimensional syneresis while $\mathrm{KI}$ induces no apparent change.

Fig. 2 shows the concentration effects of Thoulet's reagent on the precipitation of AM in varied $\mathrm{KCl}$ concentrations. By addition of $10^{-5} \mathrm{M}$ or $10^{-4} \mathrm{M}$ of Thoulet's reagent, $\mathrm{AM}$ precipitates without syneresis in the range of $\mathrm{KCl}$ concentration lower than $0.5 \mathrm{M}$ and, when $\mathrm{KCl}$ concentration is higher than $0.6 \mathrm{M}$, the solution shows only turbidity. When the concentration of Thoulet's reagent is $5 \times 10^{-4} \mathrm{M}$, the plug-formation can be observed up to $0.5 \mathrm{M} \mathrm{KCl}$ and, according to further increase of $\mathrm{KCl}$ concentration, the solution shows precipitation without syneresis and turbidity. When the concentration of Thoulet's reagent is over $10^{-3} \mathrm{M}$, the precipitation occurs with plug-formation within the whole range of examined $\mathrm{KCl}$ concentration $(0.1-1.0 \mathrm{M})$.

The grade of precipitation or turbidity becomes more and more intense with the increase of the concentration of Thoulet's reagent or the decrease of the 


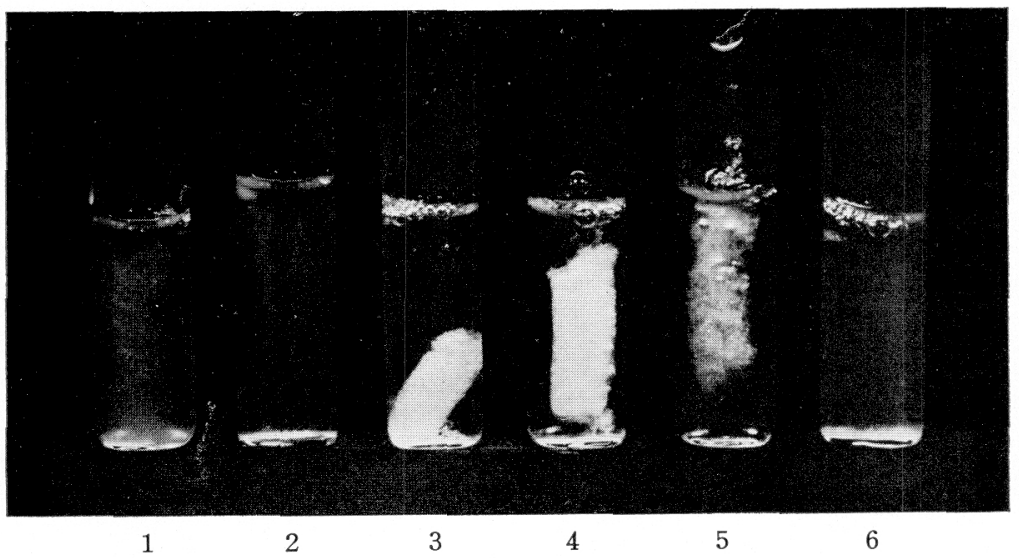

FIG. 1. Effect of Thoulet's reagent, ATP and some other salts on AM-solution.

Myosin-B: $1 \mathrm{mg} / \mathrm{cc}, \mathrm{pH}: 7$, Temperature: $20^{\circ} \mathrm{C}$.

Tube Number

1: $\operatorname{AM}(0.16 \mathrm{M} \mathrm{KCl})$

2: $\mathrm{AM}(1.0 \mathrm{M} \mathrm{KCl})$

3: $\mathrm{AM}+10^{-3} \mathrm{M} \mathrm{ATP}(0.16 \mathrm{M} \mathrm{KCl})$

4: $\mathrm{AM}+5 \times 10^{-2} \mathrm{M}$ Thoulet's reagent $(1.0 \mathrm{M} \mathrm{KCl})$

5: $\mathrm{AM}+5 \times 10^{-2} \mathrm{M} \mathrm{CuSO}_{4}(1.0 \mathrm{M} \mathrm{KCl})$

6: $\mathrm{AM}+5 \times 10^{-2} \mathrm{M} \mathrm{KI}(1.0 \mathrm{M} \mathrm{KCl})$.

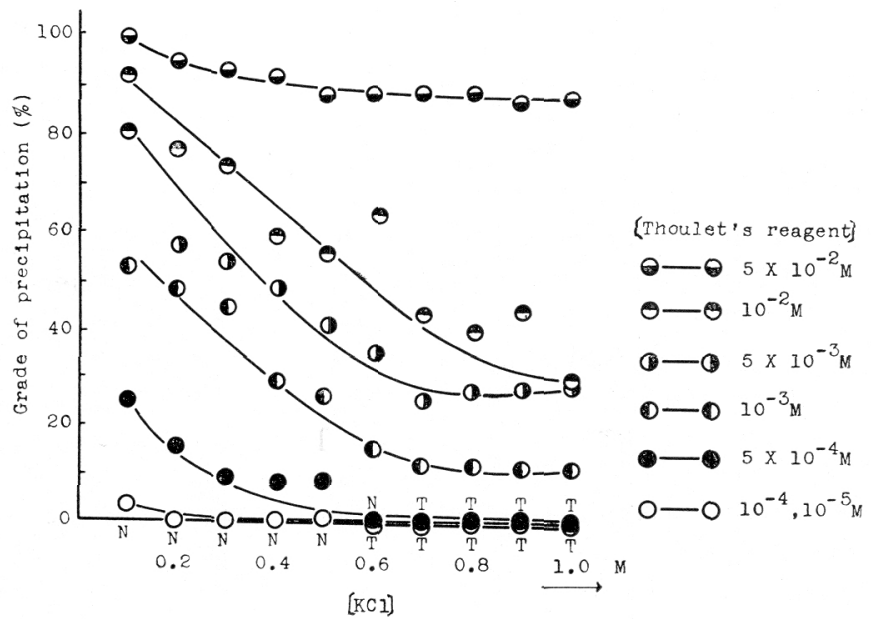

FIG. 2. The grade of precipitation of AM by varied concentrations of Thoulet's reagent at varied concentrations of $\mathrm{KCl}$.

Myosin-B: $1.04 \mathrm{mg} / \mathrm{cc}, p \mathrm{H}: 7$, Temperature: $20^{\circ} \mathrm{C}$.

$N$ : Precipitation without syneresis

$T$ : Turbidity

The other points without marks mean the syneresis with plug-formation. 
$\mathrm{KCl}$ concentration. However, when the concentration of Thoulet's reagent increases to $5 \times 10^{-2} \mathrm{M}$, the influence of the change of $\mathrm{KCl}$ concentration on the precipitation becomes not so obvious.

The precipitate of AM formed once by Thoulet's reagent could not be redissolved even when the $\mathrm{KCl}$ concentration was increased up to $2.0 \mathrm{M}$.

As shown in fig. 3, crystalline myosin shows faint flocculation under 0.066 $\mathrm{M} \mathrm{KCl}$ ( $p_{\mathrm{H}} 7$ ). Thoulet's reagent of $5 \times 10^{-2} \mathrm{M}$ induces the precipitation of crystalline myosin with plug-formation. The progress of the reaction is slow also

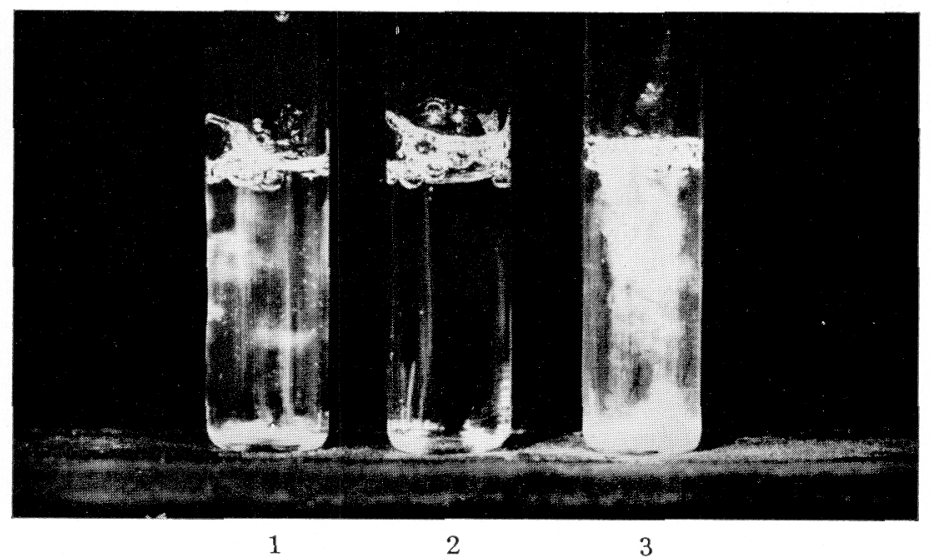

FIG. 3. Effect of Thoulet's reagent and ATP on crystalline myosin.

Myosin: $1 \mathrm{mg} / \mathrm{cc}, \mathrm{KCl}: 0.066 \mathrm{M}, \quad p \mathrm{H}: 7$, Temperature: $20^{\circ} \mathrm{C}$.

Tube Number

1: myosin alone

2: $\operatorname{myosin}+10^{-3} \mathrm{M} \mathrm{ATP}$

3: myosin $+5 \times 10^{-2} \mathrm{M}$ Thoulet's reagent.

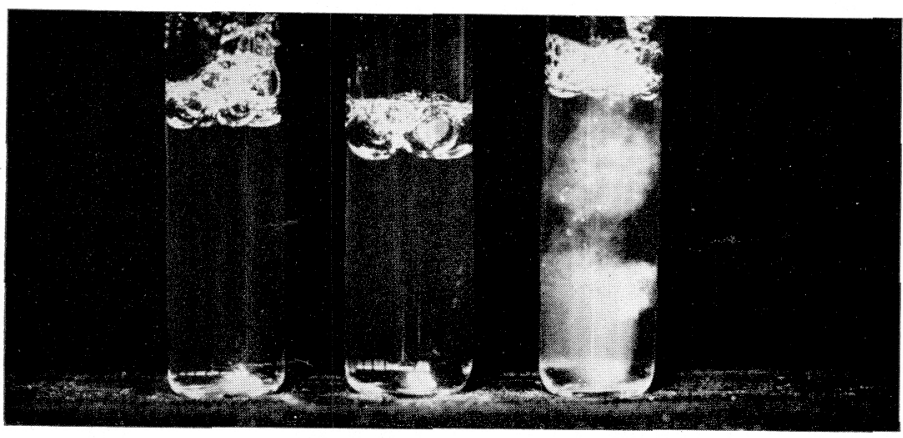

2

Fig. 4. Effect of Thoulet's reagent and ATP on L-meromyosin.

L-meromyosin: $1 \mathrm{mg} / \mathrm{cc}, \mathrm{KCl}: 0.18 \mathrm{M}, \quad p \mathrm{H}: 7$, Temperature: $20^{\circ} \mathrm{C}$. Tube Number

1: L-meromyosin alone

2: L-meromyosin $+10^{-3} \mathrm{M}$ ATP

3: L-meromyosin $+5 \times 10^{-2} \mathrm{M}$ Thoulet's reagent. 
as it takes 20-30 minutes to complete the plug-formation. On the contrary, ATP of $10^{-3} \mathrm{M}$ does not induce the precipitation of crystalline myosin but merely clearing of the solution.

As shown in fig. 4, L-meromyosin shows faint fine turbidity in $0.18 \mathrm{M} \mathrm{KCl}$ solution ( $p_{\mathrm{H}} 7$ ). Thoulet's reagent of $5 \times 10^{-2} \mathrm{M}$ induces the precipitation of $\mathrm{L}$ meromyosin with plug-formation as was observed with AM and crystalline

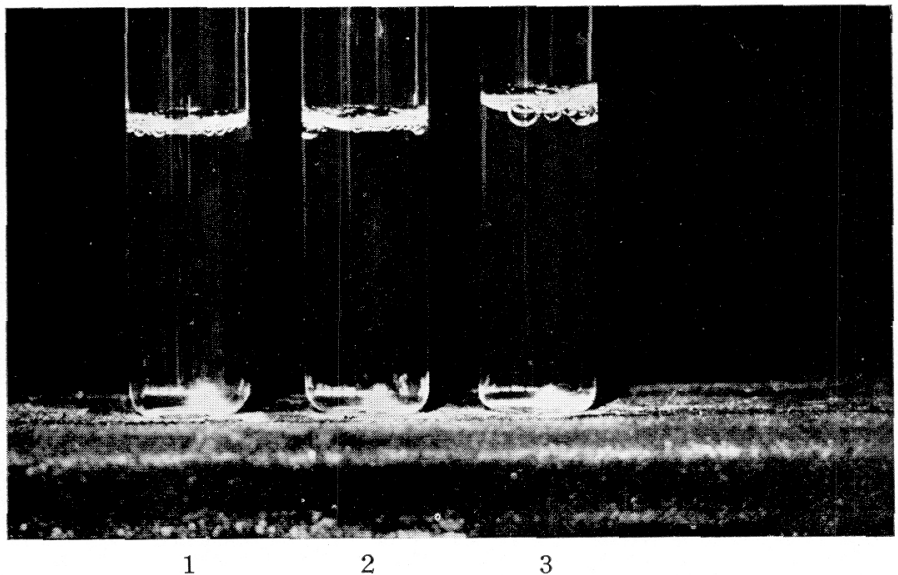

FIG. 5. Effect of Thoulet's reagent and ATP on H-meromyosin.

H-meromyosin: $1 \mathrm{mg} / \mathrm{cc}, \mathrm{KCl}: 0.04 \mathrm{M}, \quad p \mathrm{H}: 7$, Temperatnre: $20^{\circ} \mathrm{C}$. Tube Number

1: H-meromyosin alone

2: $\mathrm{H}$-meromyosin $+10^{-3} \mathrm{M}$ ATP

3: H-meromyosin $+5 \times 10^{-2} \mathrm{M}$ Thoulet's reagent.

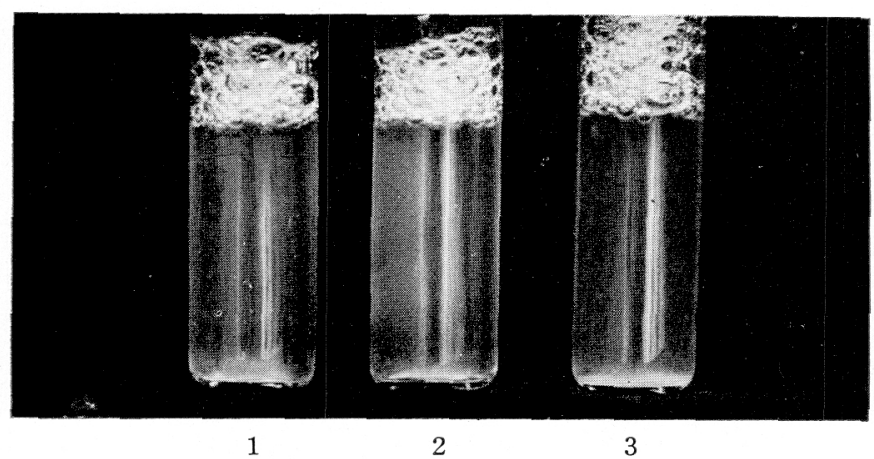

FIG. 6. Effect of Thoulet's reagent and ATP on F-actin.

F-action: $2 \mathrm{mg} / \mathrm{cc}, \mathrm{KCl}: 0.1 \mathrm{M}, p \mathrm{H}: 7$, Temperature: $20^{\circ} \mathrm{C}$. Tube Number
1: F-actin alone
2: F-action $+10^{-3} \mathrm{M} \mathrm{ATP}$
3: F-actin $+5 \times 10^{-2} \mathrm{M}$ Thoulet's reagent. 
myosin. ATP of $10^{-3} \mathrm{M}$ does not induce any change on the appearance of $\mathrm{L}$ meromyosin solution.

Under $0.04 \mathrm{M} \mathrm{KCl}(p \mathrm{H} 7)$, the solution of H-meromyosin is transparent as shown in fig. 5. Thoulet's reagent of $5 \times 10^{-2} \mathrm{M}$ or ATP of $10^{-3} \mathrm{M}$ does not induce any change on the appearance of $\mathrm{H}$-meromyosin under this condition.

As shown in fig. 6, F-actin solution is turbid under $0.1 \mathrm{M} \mathrm{KCl}(p \mathrm{H} 7)$ and very viscous. Thoulet's reagent of $5 \times 10^{-2} \mathrm{M}$ or ATP of $10^{-3} \mathrm{M}$ induces no change on the appearance of $\mathrm{F}$-actin solution under this condition. But it was observed that viscosity of $\mathrm{F}$-actin solution drops instantaneously down to about $50 \%$ of its original value by addition of $5 \times 10^{-2} \mathrm{M}$ Thoulet's reagent.

\section{DISCUSSION}

As stated above, there are considerable differences between the effect of ATP and that of Thoulet's reagent on AM and its component proteins; actin, myosin and two meromyosins.

In short, as well known, ATP can induce the contraction (syneresis) of AM only and this ATP contraction occurs merely under some limited experimental conditions. While, Thoulet's reagent induces not only syneresis of AM but that of myosin and L-meromyosin. Furthermore, the syneresis of AM induced by Thoulet's reagent is seriously different from that induced by ATP not only at the point of experimental conditions in which the syneresis occurs but also at the external appearance of the plug own.

From these data, it is suggested that Thoulet's reagent can induce not merely syneresis of actomyosin but that of fibrous proteins generally. And this point of view is supported from the other side by the fact that Thoulet's reagent shortens native or glycerinated tendon also $(6,11)$. F-actin shows no syneresis by Thoulet's reagent. However, this phenomenon may be caused by the depolymerization of actin particles by $\mathrm{KI}$ (12) contained in the reagent. Practically, the present authors observed the notable drop of viscosity of the $\mathrm{F}$-actin solution in addition of Thoulet's reagent.

Further, according to the latest observation by Kuji (13), Thoulet's reagent remarkably inhibits the ATPase activity of $\mathrm{H}$-meromyosin solution though the solution shows no change of the external appearance by the agent.

The data stated above are in a good agreement with the preceding paper of the authors (6) in which it was reported that the contraction of the glycerinated muscle fiber induced by Thoulet's reagent differs from that induced by ATP solution concerning the influence of many factors. Furthermore, it was stated that Thoulet's reagent contraction of the glycerinated muscle fiber is considered to be due to the denaturation of AM standing on the fact that contraction caused by Thoulet's reagent resembles with contraction induced by $\mathrm{CuCl}_{2}$ or by heat. This view point would be supported again by one of the present results, i.e. $\mathrm{CuSO}_{4}$ induces the syneresis of $\mathrm{AM}$ solution. Besides these, it is well known that the fibrous proteins generally contract by denaturation.

Thoulet's reagent is commonly considered to react with free amino-group of proteins (11). There exist, at present, some discussions about the group of 
AM, particulary of myosin, with which ATP reacts. However, many authors have supposed that SH group may act some rôle at the active center of ATPase $(14,15,16)$. In the preceding paper, the present authors reported that Thoulet's reagent shortening of the glycerinated muscle fiber is not influenced by the blocking of SH group caused by salyrgan while ATP contraction is notably inhibited. Salyrgan treatment per se can not induce any contraction. Still more, Thoulet's reagent induces notable syneresis of L-meromyosin solution which bears no ATPase activity and does not change its external appearance by ATP as stated above. Thus, the reactive site of AM against ATP is suggested to be different from that against Thoulet's reagent and, therefore, the difference between the mechanisms of the effects of both agents is suggested.

Concerning the interaction between AM and ATP as a model of muscular contraction, one of the most fundamental discussions in these days is whether the contraction of AM is resulted from the binding of ATP with AM or from ATP splitting caused by ATPase activity. Morales (5) introduced the name "binding deformation" for the former mechanism and "splitting deformation" for the latter. Morales supports the former mechanism, seemingly taking the phenomenon of Thoulet's reagent contraction reported by Laki et al. (3) as a base of his opinion.

Certainly, it is interesting that AM system really contracts by the presence of Thoulet's reagent and that this contraction is due to the binding of $\mathrm{K}_{2} \mathrm{HgI}_{4}$ with myosin. These facts make possible a consideration that ATP concentration is also resulted from binding of ATP and myosin as is the case in Thoulet's reagent contraction. As stated above, however, the effect of Thoulet's reagent can be considered to be different from that of ATP in nature. Then, at least, the effect of Thoulet's reagent on AM-system could not support actively the view point of "binding deformation" theory.

In reference to the above discussion, the character of the interaction between AM and ATP is considered to be rather important. According to Weber (17) and one of the present authors (18), there are many proofs by which one shall believe that ATP splitting is necessary to exhibit the contraction effect of ATP while binding reaction per se between AM and ATP corresponds to the plasticizing effect. Morales (5) quoted some data indicating that there is no parallelism between ATP splitting and contraction. However, the principal part of the phenomena seems to be understood also from the authors' assumption that ATP splitting is a condition indispensable for causing contraction but not sufficient to elicit it by itself alone (18). It may be necessary to take into consideration the physical state of AM molecule as another factor for contraction. Furthermore, by A. G. Szent-Györgyi (19), Perry (20) and Buchthal (21), many facts which are difficult to illustrate from the binding deformation theory were pointed out and these authors seem to be assuming the possibility of the splitting deformation also.

\section{SUMMARY}

1) Thoulet's reagent induces contraction (syneresis) of the solutions of actomyosin (myosin-B), crystalline myosin and L-meromyosin. 
2) The experimental conditions in which Thoulet's reagent contraction occurs are very different from those in the case of ATP.

3) These results essentially consist with the results of the authors' preceding report about glycerinated muscle fiber and Thoulet's reagent.

4) Thoulet's reagent drops the viscosity of F-actin solution and gives no influence on the external appearance of H-meromyosin solution.

5) From these results, the mechanism of the effect of Thoulet's reagent was discussed and it was concluded that this mechanism is different from that of ATP.

Thus, the view point of the preceding paper was emphasized again.

\section{REFERENCES}

1. VARGA, L. Enzymologia 14: 392, 1951.

2. Weber, H. H. Ergeb. Physiol. 36: 109, 1934.

3. LAKI, K. ET AL. Biochim. et Biophys. Acta 16: 301, 1955.

4. Bowen, W. J. ET AL. Am. J. Physiol. 185: 92, 1956.

5. Morales, M. F. ET AL. Physiol. Rev. 35: 475, 1955.

6. Nagai, T. ET AL. Jap. J. Physiol. 7: 163, 1957.

7. SzEnt-Györgyi, A. Chemistry of Muscular Contraction. New York: Acad. Press, 1947 and 1951.

8. EdSAll, J. T. J. Biol. Chem. 133: 397, 1940.

9. Szent-Györgyi, A. G. Arch. Biochem. Biophys. 42: 305, 1952.

10. Straub, F. B. Stud. Szeged. 2: 3, 1942 cit. (6).

11. PRYOR, M. Prog. Biophys. 1: 216, 1950.

12. GubA, F. Nature 165: 439, 1950.

13. KUJI, K. unpublished.

14. BAIley, K. ET AL. Biochim. Biophys. Acta 1: 506, 1947.

15. KUSCHINSKY, G. ET AL. Experientia 8: 23, 1950.

16. Kielley, W. W. et AL. J. Biol. Chem. 218: 653, 1956.

17. Weber, H. H. Adv. in Prot. Chem. 7: 161, 1952.

18. NAgAI, T. Physical Chemistry of Muscular Contraction. Tokyo: Igaku Shoin, 1956.

19. SzEnt-GyÖrgYi, A. G. Adv. in Enzymol. 16: 313, 1955.

20. Perry, S. V. Physiol. Rev. 36: 1, 1956.

21. Buchthal, F. ET AL. Physiol. Rev. 36: 503, 1956. 